\title{
Evaluation of Asymptomatic Skeletal Metastasis by 99mTc MDP Bone Scans in NSCLC Patients Attending INMAS, Rajshahi
}

\author{
${ }^{1}$ Munshi Md. Arif Hosen, ${ }^{1}$ Nasrin Begum, ${ }^{1}$ Pervez Ahmed, ${ }^{1}$ Mosharrof Hossain, ${ }^{1}$ Kabiruzzaman Shah, ${ }^{1}$ Shefaly Khatun, \\ ${ }^{1}$ Shariful Islam Chowdhury, ${ }^{2}$ Fahema Farjana Shimu, ${ }^{3}$ Julekha Sharkar and ${ }^{4}$ Shish Md. Sharkar \\ ${ }^{1}$ Institute of Nuclear Medicine and Allied Sciences, Rajshahi, ${ }^{2}$ Department of Gynae and Obstetrics, Rajshahi Medical College \\ Hospital (RMCH ), ${ }^{3}$ Department of Radiation Oncology, RMCH, ${ }^{4}$ Department of Respiratory Medicine, RMCH.
}

Correspondence address: Dr. M M Arif Hosen, Assistant Professor/SMO, INMAS, Rajshahi, E-mail- dr.arif43@gmail.com

\begin{abstract}
Background: Lung cancer is the leading cause of cancer deaths worldwide. Lung cancer is often insidious, producing no symptoms until the disease is well advanced. In approximately $\mathbf{7 - 1 0 \%}$ of cases, lung cancer is diagnosed in asymptomatic patients. Some patients are asymptomatic for skeletal metastasis and a recent prospective study showed that in staging of non-small cell lung cancer (NSCLC), failure to perform a bone scan could miss asymptomatic bone metastasis in $14-22 \%$ of patients. Radionuclide bone scintigraphy with ${ }^{99 \mathrm{~m}} \mathrm{Te}$ MDP (Technetium-99mmethylene diphosphonate) is very useful nuclear imaging tool to detect skeletal metastasis with a sensitivity of $98.2 \%$ and a specificity of $95.2 \%$. The aim of this study was to detect the asymptomatic skeletal metastasis of non-small cell lung cancer patients by $99 \mathrm{mTc}$ MDP bone scan.
\end{abstract}

Patients and methods: It is a retrospective study, carried out from January 2015 to December 2016 in Institute of Nuclear Medicine and Allied Sciences, Rajshahi. Bone scan was performed by an intravenous bolus injection of $20 \mathrm{~m} \mathrm{Ci}^{99 \mathrm{~m}} \mathrm{Tc}$ MDP. Bone phase images were taken at three hours after injection of the radiotracer.

Results: A total of 52 patients were enrolled in the study. The mean age of the patients was $57.6 \pm \mathbf{1 4 . 5}$ years (mean \pm SD) with range from 36 to 75 years and $42(80.77 \%)$ were male and 10 (19.23\%) were female. Out of 52 patients, only 06 (11.54\%) patients were diagnosed as positive and $46(88.46 \%)$ were negative for bone metastasis. Out of 06 patients with positive skeletal metastasis $04(66.67 \%)$ patients had multiple sites (two or more) and $02(33.33 \%)$ patients had solitary site of bone involvement. Common sites of metastasis were in thoracic $(83.33 \%)$ and lumbar spine $(83.33 \%)$, ribs $(50 \%)$, pelvic bones $(33.33 \%)$, long bones $(16.66 \%)$, skull $(16.66 \%)$ and other bony sites including clavicle, sternum, scapula, sacrum (16.66\%). Regarding staging prior to bone scan, out of 06 bone scan positive patients $03(50 \%)$ patients were in stage III, $02(33.33 \%)$ patients were in stage IV and $01(16.67 \%)$ patient was in stage II. On the basis of histopathology, out of 06 skeletal metastatic patients 04 $(66.67 \%)$ patients had adenocarcinoma, $01(16.67 \%)$ patient had squamous cell carcinoma and $01(16.67 \%)$ had large cell carcinoma. The exclusion of bone metastasis is important in the initial staging of non-small cell lung cancer.

Conclusion: There is debate about whether bone scans should be performed routinely or restricted to patients who present with clinical or laboratory indicators suggesting skeletal metastasis. The present study indicates that if bone scans were done only in patients reporting skeletal symptoms an important number of patients $(11.54 \%)$ would have been miss-staged due to asymptomatic bone metastasis. As bone scan is very cost effective in comparison to other imaging modalities (MRI / PET-CT), it can play a vital role in detecting asymptomatic skeletal metastasis in NSCLC patients.

Key words: NSCLC, 99mTc MDP bone scan, skeletal metastasis.

\section{INTRODUCTION}

Lung cancer is the leading cause of cancer deaths worldwide. Every year, lung cancer causes more than 1.6 million deaths; more than breast, colon and prostate cancers combined (1). Lung cancer is often insidious, producing no symptoms until the disease is well advanced. In approximately $7-10 \%$ of cases, lung cancer is diagnosed in asymptomatic patients when a chest radiograph performed for other reasons reveals the disease. At initial diagnosis, $20 \%$ of patients have localized disease, $25 \%$ of patients have regional metastasis, and $55 \%$ of patients have distant spread of disease (2). The two main types of lung cancer are small cell lung cancer (SCLC) and non-small cell lung cancer (NSCLC) $(3,4)$. NSCLC remains $80 \%-85 \%$ of all lung cancer diagnosis (5).The majorities of them present as late-stage disease with initial asymptomatic nature of early disease (6). Histologically, NSCLC is divided into adenocarcinoma, squamous cell carcinoma, and large cell carcinoma (7). NSCLC 
frequently metastasize to bone, with metastasis in bone at post-mortem in up to $36 \%$ of patients (8) and bone marrow micro-metastasis found in $22 \%-60 \%$ of individuals (9). The bone microenvironment is exposed to many growth factors and cytokines that provide a fertile place for cancer cells, making bone a preferred site of metastasis in advanced cancer. Patients with lung cancer and bone metastasis have poor prognosis with median survival times from detection of metastasis are months (8). Most of the patients with bone metastasis develop complications such as hypercalcaemia, severe bone pain, pathological fractures, spinal cord compression and bone instability. However, some patients are asymptomatic for skeletal metastasis and a recent prospective study showed that in staging of non-small cell lung cancer (NSCLC), failure to perform a bone scan could miss asymptomatic bone metastasis in $14 \%-22 \%$ of patients (10). Radionuclide bone scintigraphy with Technetium-99m methylene diphosphonate $\left({ }^{99 \mathrm{~m}} \mathrm{Tc}\right.$ MDP) is highly sensitive but usually has a low specificity: it relies on the detection of an osteoblastic reaction to suggest the presence of bone damage. In a 10-year follow-up study of Crippa et al., involving 260 patients with 1971 scans in the same institution, bone scans showed a sensitivity of $98.2 \%$, a specificity of $95.2 \%$ and a positive predictive value of $72.8 \%$, while the negative predictive value was $99.8 \%$ (11). Otherwise, sensitivity of ${ }^{99 \mathrm{~m}} \mathrm{Tc}$ scintigraphy has been reported to range from $62 \%$ to $89 \%$, with a false-positive rate as high as $40 \%$ (12); it is more sensitive and more specific than plain films and CT, while MRI was found by Algra et al. to be superior to bone scan in evaluating vertebral metastasis (13). The aim of this retrospective study was to detect the asymptomatic skeletal metastasis of non-small cell lung cancer patients by ${ }^{99 \mathrm{~m}} \mathrm{Tc}$ MDP bone scan.

\section{PATIENTS AND METHODS}

This retrospective study was carried out from January 2015 to December 2016 in Institute of Nuclear Medicine and Allied Sciences (INMAS), Rajshahi. A total 230 lung cancer patients were referred for radionuclide bone scan. Patient's clinical records and bone scan reports were reviewed retrospectively. Inclusion criteria include patients with histologically confirmed non-small cell lung cancer without prior evidence of clinical or radiological skeletal metastasis. Patients with any skeletal symptoms including bone pain, trauma, metabolic bone disease, arthritis or arthropathy were excluded from the study. A total of 52 patients were recruited in this study those had the inclusion criteria out of 230 lung cancer patients. Scintigraphy were performed using digital dual head gamma camera (e-cam series, Siemens from Germany) with a low-energy high resolution parallel hole collimator after an intravenous bolus injection of $20 \mathrm{~m}$ $\mathrm{Ci}{ }^{99 \mathrm{~m}} \mathrm{Tc} \mathrm{MDP}$. Delayed bone images were taken at three hours after injection of the radiotracer and the scan time was about 15-18 minutes for a whole body scan. Whole body scan in anterior and posterior projections were obtained. All bone scans were reviewed by two well-trained nuclear medicine physicians.

\section{RESULTS}

A total of 52 patients were enrolled in the study. The mean age of the patients was $57.6 \pm 14.5$ years (mean $\pm \mathrm{SD}$ ) with range from 36 to 75 years and $42(80.77 \%)$ were male and $10(19.23 \%)$ were female. Out of 52 patients, only 06 (11.54\%) patients were diagnosed as positive and 46 $(88.46 \%)$ were negative for bone metastasis (Figure 1).

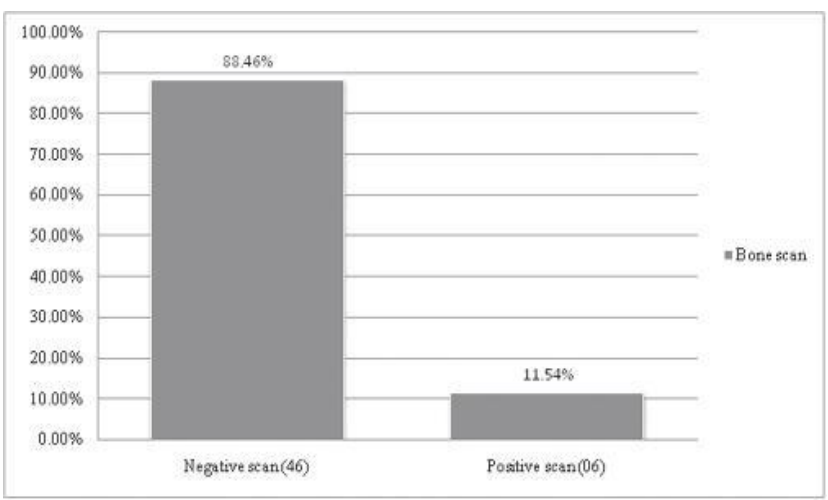

Figure 1: Distribution of bone scan findings in frequency and percentage $(n=52)$

Out of 06 patients with positive skeletal metastasis 04 (66.67\%) patients had multiple sites (two or more) and $02(33.33 \%)$ patients had solitary site of bone involvement (Figure 2). 


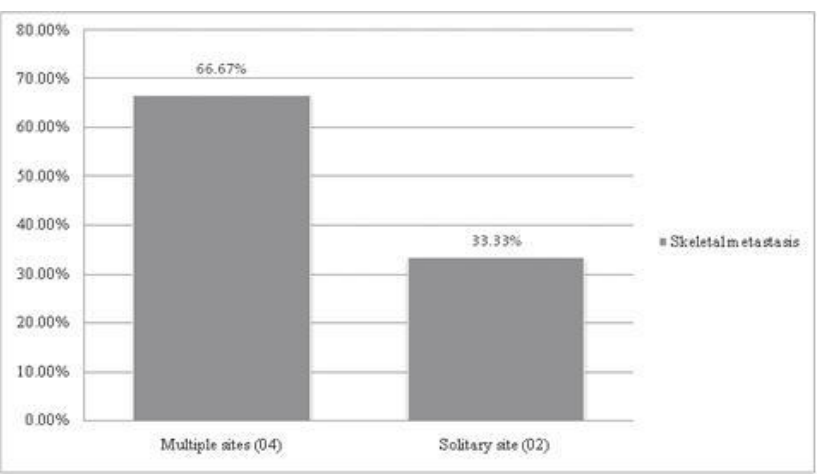

Figure 2: Distribution of skeletal metastasis in frequency and percentage by number of bony site involvement $(n=06)$

Common sites of involvement were in thoracic $(83.33 \%)$ and lumbar spine $(83.33 \%)$, ribs $(50 \%)$, pelvic bones $(33.33 \%)$, long bones $(16.66 \%)$, skull $(16.66 \%)$ and other bony sites including clavicle, sternum, scapula, sacrum (16.66\%) (Figure 3).

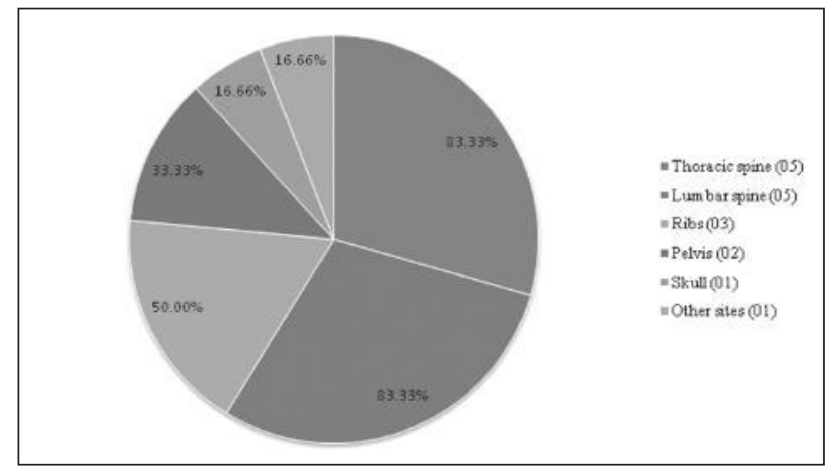

Figure 3: Distribution of skeletal metastasis in frequency and percentage by anatomical sites $(n=06)$

Regarding staging prior to bone scan, out of 06 bone scan positive patients $03(50 \%)$ patients were in stage III, $02(33.33 \%)$ patients were in stage IV and 01(16.67\%) patient was in stage II (Figure 4).

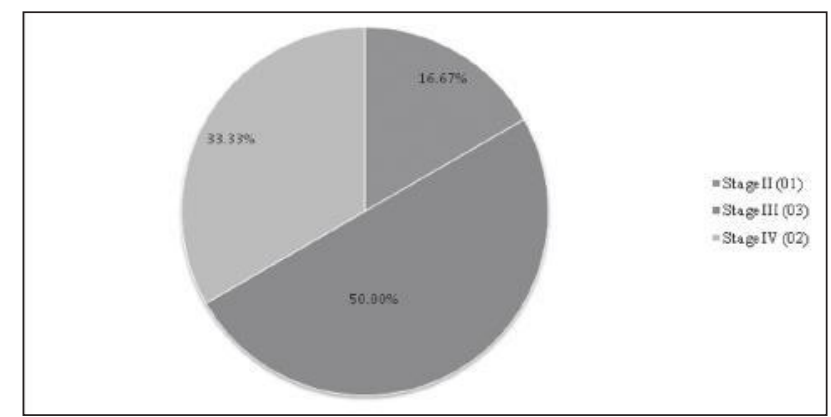

Figure 4: Distribution of staging in frequency and percentage prior to bone scan in skeletal metastatic patients $(n=06)$
On the basis of histopathology, out of 06 skeletal metastatic patients $04(66.67 \%)$ patients had adenocarcinoma, $01(16.67 \%)$ patient had squamous cell carcinoma and $01(16.67 \%)$ had large cell carcinoma (Figure 5).

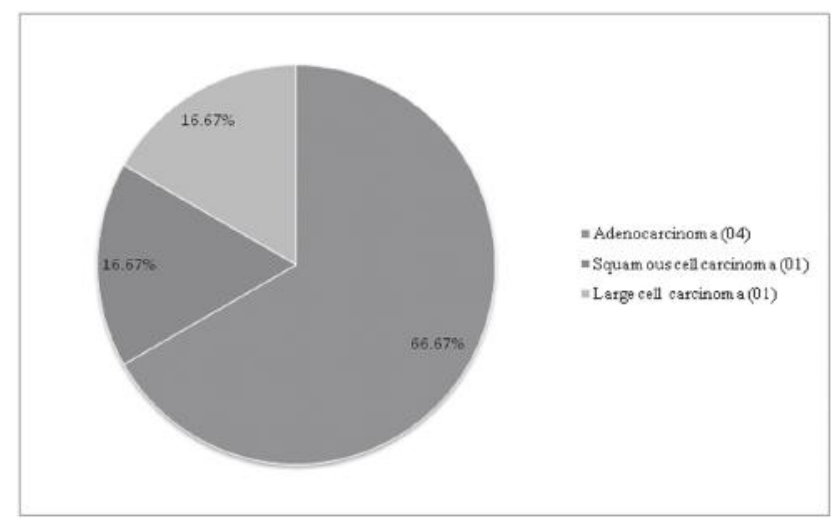

Figure 5: Distribution ofhistopathological types in frequency and percentage in skeletal metastatic patients $(n=06)$

Common sites of involvement were in thoracic $(83.33 \%)$ and lumbar spine (83.33\%), ribs $(50 \%)$, pelvic bones $(33.33 \%)$, long bones $(16.66 \%)$, skull $(16.66 \%)$ and other bony sites including clavicle, sternum, scapula, sacrum (16.66\%) (Figure 3). Regarding staging prior to bone scan, out of 06 bone scan positive patients $03(50 \%)$ patients were in stage III, $02(33.33 \%)$ patients were in stage IV and 01(16.67\%) patient was in stage II (Figure 4). Figure 4: Distribution of staging in frequency and percentage prior to bone scan in skeletal metastatic patients $(\mathrm{n}=$ 06) On the basis of histopathology, out of 06 skeletal metastatic patients $04(66.67 \%)$ patients had adenocarcinoma, $01(16.67 \%)$ patient had squamous cell carcinoma and $01(16.67 \%)$ had large cell carcinoma (Figure 5). Figure 5: Distribution of histopathological types in frequency and percentage in skeletal metastatic patients $(n=06)$

\section{DISCUSSION}

Lung cancer (both SCLC and NSCLC) is the third most common form of cancer to spread to bone. About $30-40 \%$ of patients with lung cancer developed bone metastasis during the course of their disease; the 
median survival time of patients with this secondary lesion is 7 months (14). These metastases are associated with significant morbidity, loss of functional independence and reduction in quality of life (15). Bone metastasis accounts for 350,000 cancer patient deaths each year (16), and in lung cancer is associated with increased social costs due to medical care, hospitalization days and cost of treatment (17). Bone scintigraphy has been a perfect method for evaluation of bone metastasis and cancer staging for several decades and it is still a procedure of choice in many of these clinical settings $(18,19)$. In this study, out of 52 NSCLC patients $11.54 \%$ (06 patients) had positive bone scan though they were asymptomatic for bone metastasis. Several studies carried out to evaluate asymptomatic skeletal metastasis in lung cancer patients. Hooper et al. found abnormal bone scan in 4\% asymptomatic patients (20). Kelly et al. obtained positive bone scan in 14\% asymptomatic patients (21). Schirrmeister et al. found positive bone scan in 10\%-14\% asymptomatic NSCLC patients (10). Iordanidou et al. found skeletal metastasis in $16.3 \%$ asymptomatic NSCLC patients (22). Afrin et al. found $44 \%$ positive bone scan in asymptomatic lung cancer patients (23). The present study showed in positive bone scan patients $66.67 \%$ had multiple sites (two or more) and $33.33 \%$ patients had solitary site of bone involvement. Common sites of metastases were in thoracic $(83.33 \%)$ and lumbar spine $(83.33 \%)$, ribs $(50 \%)$, pelvic bones $(33.33 \%)$, long bones $(16.66 \%)$, skull (16.66\%) and other bony sites including clavicle, sternum, scapula, sacrum (16.66\%). The findings were almost similar with other studies, Afrin et al. obtained high incidence of bone metastasis from lung cancer was seen in the axial skeleton like vertebra (54\%), ribs (47\%), and pelvis (22\%) (10), Vahid Reza et al. obtained the most frequently involved area was the spine, followed by ribs and pelvic bones (24). In a retrospective study of 259 non-small cell lung cancer (NSCLC) patients, the most common site of skeletal metastases was the spine in $50 \%$ of patients, followed by the ribs $(27.1 \%)$, ilium $(10 \%)$, sacrum $(7.1 \%)$, femur $(5.7 \%)$ and humerus, scapula and sternum $(2.9 \%)(25)$. Prognosis was worse in patients with metastasis to the appendicular bone than in patients with metastasis only on an axial bone (26). Regarding staging prior to bone scan, out of 06 bone scan positive patients $03(50 \%)$ patients were in stage III, 02 (33.33\%) patients were in stage IV and 01(16.67\%) patient was in stage II. So staging was changed to stage-IV disease for $66.67 \%$ patients and no stage-I patient had skeletal metastasis in the study. Treatment is palliative in stage-IV lung cancer patients, thus sparing unnecessary surgery or neoadjuvant therapy (10). In our study, On the basis of histopathology, in bone scan positive patients $66.67 \%$ had adenocarcinoma, $16.67 \%$ had squamous cell carcinoma and $16.67 \%$ had large cell carcinoma. Serdar Erturan et al. found that adenocarcinoma was the most common cell type in NSCLC patients with bone metastasis (39\%) (27).

\section{CONCLUSION}

The exclusion of bone metastasis is important in the initial staging of non-small cell lung cancer. There is debate about whether bone scans should be performed routinely or restricted to patients who present with clinical or laboratory indicators suggesting skeletal metastases. The present study indicates that if bone scans were done only in patients reporting skeletal symptoms an important number of patients (11.54\%) would have been miss-staged due to asymptomatic bone metastasis. As bone scan is very cost effective in comparison to other imaging modalities (MRI / PET-CT), it can play a vital role in detecting asymptomatic skeletal metastasis in NSCLC patients. Thus the authors suggest to perform bone scintigraphy for initial staging of all patients of NSCLC.

\section{REFERENCES}

1. LCSM Lung Cancer Facts. Access Date: Oct. 7, 2015: http://lcsmchat.com/lung-cancer-facts/\#edn12.

2. Corner J, Hopkinson J, Fitzsimmons D, Barclay S, Muers M. Is late diagnosis of lung cancer inevitable? Interview study of patients' recollections of symptoms before diagnosis. Thorax 2005;60(4):314-9. 
3. Navada S, Lai P, Schwartz AG, Kalemkerian GP. Temporal trends in small cell lung cancer: analysis of the national Surveillance Epidemiology and End-Results (SEER) database [abstract 7082] J Clin Oncol 2006;24(18S)suppl:384S.

4. Sher T, Dy GK, Adjei AA. Small cell lung cancer. Mayo Clin Proc 2008;83(3):355-67.

5. D'Addario $G$, Fruh $M$, Reck $M$ et al. Metastatic non-small-cell lung cancer: ESMO Clinical Practice Guidelines for diagnosis, treatment and follow-up. Ann Oncol 2010; 21Suppl 5v116-v119.

6. Stinchcombe TE, Lee CB, Socinski MA. Current approaches to advanced-stage non- small-cell lung cancer: first-line therapy in patients with a good functional status. Clin Lung Cancer 2006; 7Suppl 4S111-S117.

7. http://emedicine.medscape.com/article/279960-overview.

8. Coleman RE. Clinical features of metastatic bone disease and risk of skeletal morbidity. Clin Cancer Res 2006;12:6243-49.

9. Coello MC, Luketich JD, Litle VR, Godfrey TE. Prognostic significance of micrometastases in non-small-cell lung cancer. Clin Lung Cancer 2004; 5: 214-25.

10. Schirrmeister $\mathrm{H}$, Arslandemir $\mathrm{C}$, Glatting $\mathrm{G}$ et al. Omission of bone scanning according to staging guidelines leads to futile therapy in non-small cell lung cancer. Eur J Nucl Med Mol Imaging 2004; 31: 964-68.

11. Crippa F, Seregni E, Agresti R, Bombardieri E and Buraggi GL. Bone scintigraphy in breast cancer: a ten years follow up study. J Nucl Biol Med 1993; 37:57-61.

12. Quinn DL, Ostrow LB, Poerter DK et al. Staging of non-small cell bronchogenic carcinoma: relationship of the clinical evaluation to organ scans. Chest 1986; 89:270-75.

13. Algra PR, Bloem JL, Tissing $H$ et al. Detection of vertebral metastases: comparison between MRI and bone scintigraphy. Radiographics 1991; 11: 219-32

14. Coleman R. Metastatic bone disease: clinical features, pathophysiology and treatment strategies. Cancer Treat Rev 2001;27:165-76.

15. Berenson J, Rajdev L, Broder M. Managing bone complications of solid tumors. Cancer Biol Ther 2006; 5:1086-89.

16. Mundy G. Metastasis to bone: causes, consequences and therapeutic opportunities. Nat Rev Cancer 2002;2:584-93.
17. Botteman M, Foley I, Marfatia A, Brandman J, Langer C. Economic value of Zoledronic acid versus placebo in the treatment of skeletal metastases in patients with lung cancer: the case of the United Kingdom (UK). J Clin Oncol 2007;25:6617.

18. Morgan JWM, Adcock KM, Donohue RE. Distribution of skeletal metastases in prostatic and lung cancer. Urology 1990;36:31-34.

19. Wilson MA, Calhoun FW. The distribution of skeletal metastases in breast and pulmonary cancer: concise communication. J Nucl Med 1981;22:594-97.

20. Hooper RG, Beecheler CR, Johnson MC. Radioiodine scanning in the initial staging of bronchogenic carcinoma. Am Rev Resp Dis 1978;118:279-86.

21. Kelly RJ, Cason RJ, Ferrie CB. Efficacy of radionuclide scanning in patient with lung cancer. JAMA 1979;242:2855-57.

22. Iordanidou L, Trivizaki E, Saranti S, Georgakopoulos A, Bolanos N, Baltagiannis N, Koutsiouba P. Is there a role of whole body bone scan in early stages of non-small cell lung cancer patients? J BUON. 2006 Oct-Dec;11(4):491-7.

23. Afrin R, Haque FS, Biswas SK, Hossain S, Roy SK, Jahan M. Role of Whole-Body Tc 99m MDP Bone Scintigraphy for Evaluating Skeletal Metastasis in Patients with Lung Cancer. Bangladesh Med Res Counc Bull 2016;42:132-136

24. Vahid Reza, DabbaghKakhki, KazemAnvari, RaminSadeghi, Anooshe-Sadat Mahmoudian, Maryam Torabian-Kakhki. Pattern and distribution of bone metastases in common malignant tumors. Nuclear Medicine Review 2013;16(2):66-69.

25. Tsuya A, Kurata T, Tamura K, Fukuoka M. Skeletal metastases in non- small cell lung cancer: a retrospective study. Lung Cancer 2007;57:229-32.

26. Sugiura H, Yamada K, Sugiura T, Hida T, Mitsudomi T. Predictors of Survival in patients with bone metastasis of Lung Cancer. Clin Orthop Relat Res 2008;466:729-36.

27. Serdar Erturan, Mustafa Yaman, Gunay Aydın, IsılUzel, BenanMusellim, KamilKaynak. The Role of Whole-Body Bone Scanning and Clinical Factors in Detecting Bone Metastases in Patients With Non-small Cell Lung Cancer. J Chest 2005;127(2):449-54. 